\title{
论文
}

\section{中国电网停电事故时间间隔的统计及分布特性 分析}

\author{
于群 ${ }^{1}$, 屈玉清 ${ }^{*}$, 曹娜 ${ }^{1}$, 易俊 ${ }^{2}$
}

1. 山东科技大学电气与自动化工程学院, 青岛 266590;

2. 中国电力科学研究院有限公司电力系统研究所, 北京 100085

* E-mail: 01quyuqing@163.com

收稿日期: 2018-04-17; 接受日期: 2018-10-15; 网络版发表日期: 2018-11-27

国家电网公司2018年科技项目(基于多沙堆理论的互联电网停电事故预警技术及系统研发)和山东科技大学研究生科技创新项目(编号: SDKDYC180233)资助

摘要为研究中国电网停电事故的复杂动态特性, 分析了中国电网停电事故时间间隔的分布特性. 首先, 对中国 电网1981 2014年的停电事故的时间间隔进行了统计分析和正态性检验，指出了中国电网停电事故的时间间隔近 似满足幕律分布. 其次, 利用重标方差 (rescaled variance, V/S) 方法对中国电网停电事故的时间间隔序列进行了相 关性分析，指出了中国电网停电事故的时间间隔序列具有长程正相关性和统计自相似性. 最后, 给出了电网停电 事故时间间隔分布特性的意义.

关键词停电事故, 时间间隔, 幕律分布, V/S 方法, 相关性, 分布特性

\section{1 引言}

在当代社会的发展进程中，电力承担着重要的作 用，世界各国高度重视电网的安全运行. 然而，国内外 电网的停电事故时有发生，给各国带来了巨大影响和 损失, 如: 2003年美国东部和加拿大大停电事故, 损失 负荷 $61.8 \mathrm{GW}$ ，造成经济损失300亿美元；2009年巴西 大停电事故，损失负荷 $24 \mathrm{GW} ; 2011$ 年日本大停电事 故，损失负荷 $22 \mathrm{GW} ; 2012$ 年印度大停电事故，损失负 荷50 GW $\mathrm{GW}^{[1]}$ 等，而这些大停电事故大多是由连锁故障 导致的 ${ }^{[2-4]}$. 这些连锁故障及大停电事故的发生表明了
电网停电事故内在动力学行为上的复杂性, 据此可用 复杂性科学的方法研究电力系统的大停电事故, 这就 为预防大停电事故的发生和探索大停电事故的机理提 供了新的方法和思路.

近年来，国内外学者利用复杂系统的自组织临界 理论研究了大停电事故的发生机理, 取得了很多研究 成果. 文献[5 11]分析美国电网停电规模满足幂律分 布, 揭示了美国电网具有自组织临界特性. 文献 $[12,13]$ 分析中国电网1981 2002年的停电事故损失负 荷的频度与规模之间满足幂律关系, 揭示了中国电网 具有自组织临界特性. 文献[14]基于自组织临界理论

引用格式: 于群, 屈玉清, 曹娜, 等. 中国电网停电事故时间间隔的统计及分布特性分析. 中国科学: 技术科学, 2019, 49: 55-62 Yu Q, Qu Y Q, Cao N, et al. Statistics and distribution characteristics analysis of time interval of blackout in Chinese power grid (in Chinese). Sci Sin Tech, 2019, 49: 55-62, doi: 10.1360/N092018-00103 
提出了反映电网连锁故障的演化模型. 上述研究结论 都是基于电网停电事故损失规模得到的. 文献[15]分 析区域配电网停电故障在时域上具有幂律分布特性和 长程自相关性，得出了区域配电网故障具有自组织临 界性的结论, 但其未对输电网的停电事故进行研究. 在电网的停电事故中, 输电网的连锁故障往往是导致 大停电事故的主因，然而，目前针对输电网停电事故 的时间行为分布特性的研究却较少, 但是, 研究电网 停电事故的时间行为的分布特性，可进一步揭示电网 停电事故的内在动力学机理, 对预防大停电事故的发 生和评估电网风险具有重要意义.

表征电网停电事故时间行为的主要变量有事故发 生的时间、事故持续时间和事故时间间隔等，而电网 停电事故发生的具体时间是难以预测的，早期事故的 持续时间是记录不全的，但事故发生的时间间隔是可 以统计分析的，时间间隔既能度量事件的时间行为, 也能反映事件的内在演化规律. 据此，可以通过分析 电网停电事故的时间间隔探讨电网停电事故的时间行 为的分布特性.

在研究电网停电事故的时间间隔方面, 文献 $[6,8,16]$ 研究指出美国电网停电事故的时间间隔近似 满足指数分布. 文献[17]研究改进了美国电网停电事 故时间间隔的指数分布. 文献[10]研究分析了美国电 网在1984 2006年期间的停电事故时间间隔数据，指 出停电事故时间间隔数据分布与指数分布之间存在一 定的差异. 文献[11]在文献[8]的研究基础上对指数速 率进行了假设，指出了停电事故时间间隔序列与非均 匀的泊松过程一致，但未对假设进行有效的验证. 文 献[18]分析广东电网故障时间间隔的统计分布及非线 性特征, 指出了广东电网故障时间间隔近似满足幂律 分布，具有长程正相关性和自相似性，但其未涉及全 国电网故障时间行为的分布特性.

基于上述研究情况会引人思考, 中国电网停电事 故的时间行为的分布特性是什么? 其分布特性对电网 有何意义? 为解决这些问题, 本文以中国电网停电事 故的时间间隔数据为研究对象, 探讨了中国电网停电 事故的时间行为的分布特性. 首先，统计分析了中国 电网停电事故时间间隔序列的频度-标度关系和概率 分布, 并运用Kolmogorov-Smirnov(K-S)方法对数据进 行了正态性检验. 然后, 利用 $\mathrm{V} / \mathrm{S}$ 方法分析了中国电网 停电事故时间间隔序列的相关性. 最后给出了中国电
网停电事故时间间隔分布特性的意义.

\section{2 中国电网停电事故时间间隔的统计分析 及正态性检验}

根据国家电力调度通信中心和国家电网公司安全 监察部等单位发布的数据 ${ }^{[19 ~ 29]}$, 本文统计形成了 1981 2014年的中国电网(不含台湾省)的停电事故数 据序列, 其中, 有损失负荷记录的停电事故共计 277 次, 本文以这277次停电事故的时间间隔为研究对象. 需要 说明的是, 本文统计的这些停电事故主要包括由继电 保护装置和安全自动装置异常引发的事故、自然灾害 引发的事故、一次设备故障引发的事故, 以及人员责 任和外力人为破坏等电网事故.

\section{1 电网停电事故时间间隔序列的统计分析}

根据统计得到的停电事故数据序列, 可得中国电 网停电事故时间序列, 如图1所示.

在图1中, 每条垂直的坚线表示一次电网停电事 故, 纵坐标为停电事故的损失负荷 (单位: MW), 横坐 标为停电事故发生的时间(单位: 天), 取第一次停电事 故发生的时间为起点. 由图1可以看出, 中国电网停电 事故的时间间隔有大有小, 有时连续发生(时间间隔为 零天), 有时间隔一段时间才发生(时间间隔大于等于 1 天), 由此可知中国电网停电事故的时间间隔大小不成 比例, 不是线性的而是呈现非线性特征, 需要进一步研 究中国电网停电事故时间间隔的分布特性.

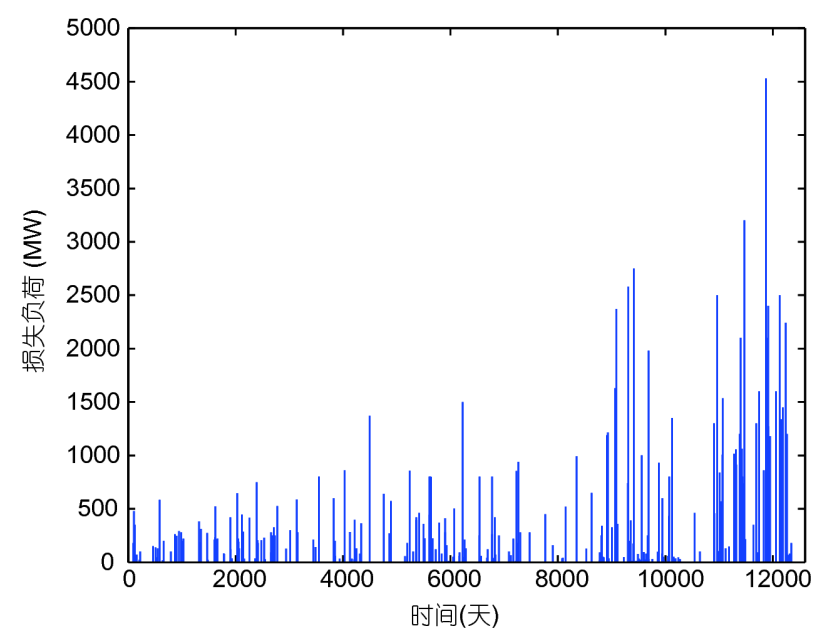

图 1 (网络版彩图)中国电网停电事故时间序列 
将1981 2014年的中国电网停电事故按照事故发 生时间的前后顺序排序, 求出相邻2次停电事故的时间 间隔 $\delta$ (单位: 天). 由 277 次停电事故可得到含有 276 个 数据的停电事故时间间隔序列.

对中国电网停电事故的时间间隔序列进行标度频度分析 ${ }^{[12]}$, 取时间间隔标度的间隔长度为 10 天, 统 计事故时间间隔的频度, 结果如表1所示.

由表 1 知，停电事故时间间隔在 250 天以上的占 $3.26 \%$, 在 150 天以上的占 $6.16 \%$, 在 100 天以上的占 $13.04 \%$, 在 50 天以上的占 $26.82 \%$, 则 $73.18 \%$ 的停电事 故的时间间隔在 50 天内. 这初步表明中国电网发生的 停电事故大多数是在短时间内 ( $0 \sim 50$ 天) 集中爆发, 少 数在间隔较长的时间(150天)后发生. 由此可知, 中国 电网发生的停电事故在时间行为上具有阵发和厚尾 特点.

以时间间隔的标度为横坐标, 其频度为纵坐标, 将 表1中的数据绘制在双对数坐标下, 如图 2 所示. 由图 2 可以看出, 中国电网停电事故时间间隔的标度-频度之 间存在幂律关系.

为使分析结果合理、有效, 避免最小二乘拟合方 法分析数据幂律产生偏差, 本文使用文献[30]所述方 法分析和检验中国电网停电事故时间间隔序列的 幂律.

$n$ 代表样本数量; $x_{\min }$ 是幂律行为的下界; $\alpha$ 是幂指 数的估计值; $n_{\mathrm{tai}}$ 是对拟合参数不确定度估计的标准偏

表 1 中国电网停电事故时间间隔频度

\begin{tabular}{cc||cc}
\hline 标度 $r$ (天) & 频度 $N$ (次) & 标度 $r($ 天 $)$ & 频度 $N$ (次) \\
\hline$\geq 10$ & 203 & $\geq 140$ & 20 \\
$\geq 20$ & 149 & $\geq 150$ & 17 \\
$\geq 30$ & 108 & $\geq 160$ & 17 \\
$\geq 40$ & 93 & $\geq 170$ & 15 \\
$\geq 50$ & 74 & $\geq 180$ & 12 \\
$\geq 60$ & 66 & $\geq 190$ & 12 \\
$\geq 70$ & 51 & $\geq 200$ & 11 \\
$\geq 80$ & 46 & $\geq 210$ & 10 \\
$\geq 90$ & 40 & $\geq 220$ & 10 \\
$\geq 100$ & 36 & $\geq 230$ & 10 \\
$\geq 110$ & 28 & $\geq 240$ & 9 \\
$\geq 120$ & 23 & $\geq 250$ & 9 \\
$\geq 130$ & 21 & & \\
\hline
\end{tabular}

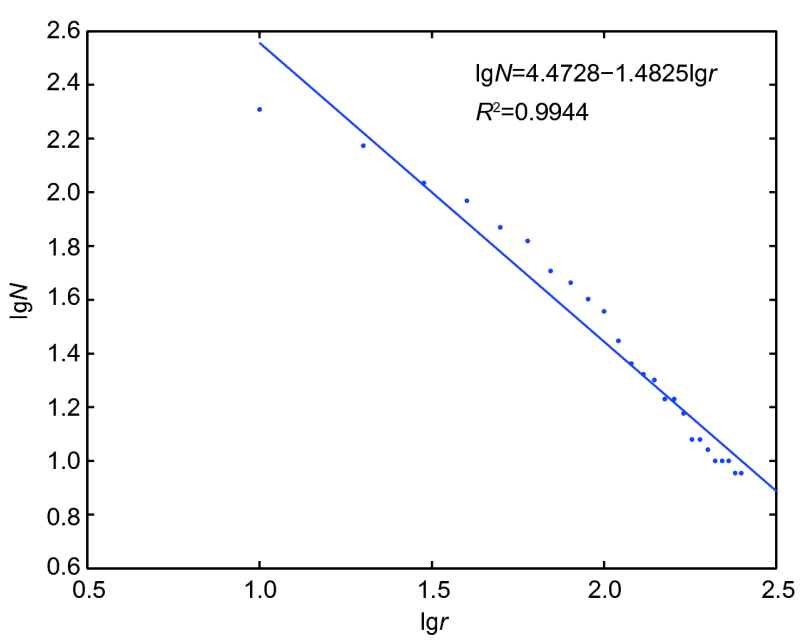

图 2 (网络版彩图)中国电网停电事故时间间隔标度频度双 对数图

差; $p$ 是量化幂律假说的合理性, 当 $p>0.1$ 时, 表示幂律 假说是合理的, 有理由相信数据满足幂律分布; 当 $p \leq 0.1$ 时, 表示拒绝幂律假说, 数据不满足幂律分布. 中国电网停电事故时间间隔序列的幕律模型参数选取 $n=276, x_{\min }=88, \alpha=2.93, n_{\text {tail }}=53, p=0.12$. 其累计概率分 布如图3所示.

由上述可知, $p>0.1$, 据此可认为中国电网停电事 故时间间隔序列的幂律分布具有合理性. 此外, 为排除 其他分布(如泊松分布或指数分布)比幂律分布更适合 的可能性, 利用似然比检验计算了幂律分布与泊松分 布及幂律分布与指数分布的对数似然比(LR).

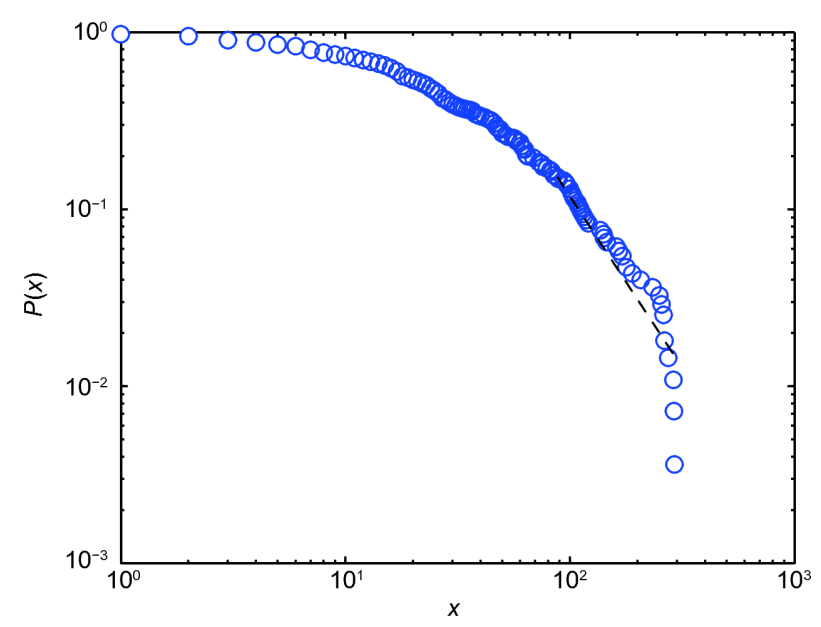

图 3 (网络版彩图)中国电网停电事故时间间隔的累计概率 分布 
中国电网停电事故时间间隔序列的幂律分布与泊 松分布 $(L R=4.32)$ 及幂律分布与指数分布的 $(L R=1.19)$ $L R$ 都大于 0 ，说明幕律分布更适合，且泊松分布 $(p=0.02)$ 及指数分布 $(p=0.05)$ 的 $p$ 值都小于 0.1 , 据此可 拒绝中国电网停电事故时间间隔序列遵循泊松分布或 指数分布的假说.

基于上述分析，可知中国电网停电事故的时间间 隔既不满足泊松分布，也不满足指数分布，而是近似 满足幂律分布.

\section{2 电网停电事故时间间隔序列的K-S正态性检验}

为准确描述中国电网停电事故时间间隔序列的统 计分布特性，运用K-S方法检验了中国电网停电事故 时间间隔数据的正态性. 选取样本数为 276 , 由统计结 果可知, 电网停电事故时间间隔序列的均值为 44.39 天, 最大值为 292 天, 最小值为 0 天, 标准差为 58.63 , 偏度为 2.395 , 大于 0 , 峰度为 8.942 , 大于 0 , 据此可知, 电网停电 事故时间间隔数据表现为尖峰、厚尾分布, 结合图 2 和 3 可知, 中国电网停电事故的时间间隔序列近似满足幂 律分布, 而不是正态分布, 可初步确定中国电网具有复 杂系统的一般动力学特征.

此外，为更直观地观察中国电网停电事故时间间 隔的数据分布，由中国电网停电事故时间间隔数据序 列可得其分布图, 结果如图4所示.

由图4可知, 中国电网停电事故时间间隔的数据分 布不满足正态分布, 与K-S正态性检验结果相符.

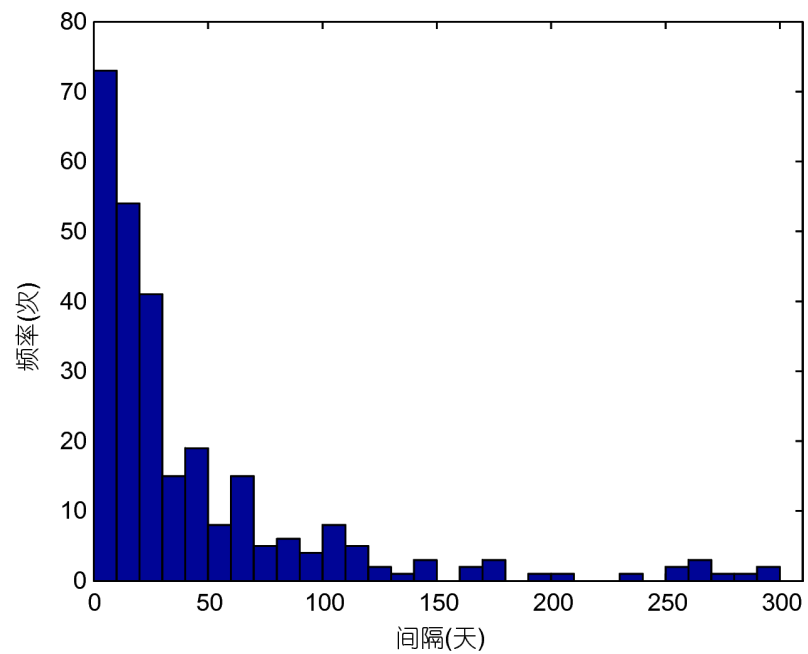

图 4 (网络版彩图)中国电网停电事故时间间隔分布图
根据文献[14]对幂律分布和长程相关的研究，可 知，能利用Hurst指数理论分析中国电网停电事故时间 间隔序列的长程相关性，常用的分析时间序列长程相 关性的方法有 $\mathrm{R} / \mathrm{S}$ (rescaled range analysis)方法和 $\mathrm{V} / \mathrm{S}$

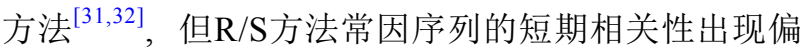
差，因此本文采用 $\mathrm{V} / \mathrm{S}$ 方法分析中国电网停电事故时 间间隔序列的长程相关性.

\section{$3 \mathrm{~V} / \mathrm{S}$ 分析方法}

V/S 分析方法是由Giraitis等人 ${ }^{[33]}$ 在2003年提出的.

\section{$3.1 \mathrm{~V} / \mathrm{S}$ 分析方法基本原理}

对时间序列 $\left\{x_{1}, x_{2}, \ldots, x_{N}\right\}$, 其中 $N$ 为样本总数, 其 子序列为 $\left\{x_{1}, x_{2}, \ldots, x_{n}\right\}, n(2 \leq n \leq N)$ 是每个子序列观测 值的个数.

$\mathrm{V} / \mathrm{S}$ 方法的分析统计量为

$V_{s}(n)=\frac{\left[\sum_{k=1}^{n}\left(\sum_{t=1}^{k}\left(x_{t}-\bar{x}\right)\right)^{2}-\frac{1}{n}\left(\sum_{k=1}^{n} \sum_{t=1}^{k}\left(x_{t}-\bar{x}\right)\right)^{2}\right]}{n^{2} S_{n, q}^{2}}$,

式中, $\bar{x}=\frac{1}{n} \sum_{t=1}^{n} x_{t}$ 为序列的均值.

$S_{n, q}^{2}=\frac{1}{n} \sum_{t=1}^{n}\left(x_{t}-\bar{x}\right)^{2}+2 \sum_{t=1}^{q} w_{t}(q) \gamma_{t}$,

式中, $w_{t}(q)=1-\frac{t}{q+1}, q<n$.

$\gamma_{t}=\frac{\sum_{i=1}^{n-t}\left(x_{i}-\bar{x}\right)\left(x_{i+t}-\bar{x}\right)}{n}$.

取不同的 $n \quad(2 \leq n \leq N)$ 值，求得不同子区间上的 $V_{s}(n)$, 存在以下关系:

$\lg \left[V_{s}(n)\right]=\lg k+2 H \lg n$,

式中, $k$ 为统计常数, $H$ 为 $\mathrm{V} / \mathrm{S}$ 方法的Hurst指数, Hurst指 数的意义及其显著性检验方法见文献[34].

\section{$3.2 \mathrm{~V} / \mathrm{S}$ 分析方法的稳定性检验}

类似于Hurst对 $\mathrm{R} / \mathrm{S}$ 构建的 $V$-统计量曲线, $\mathrm{V} / \mathrm{S}$ 分析 构造 $V_{n}{ }^{*}$ 统计量, 用 $V_{n}{ }^{*}$ 统计量曲线来检验 $\mathrm{V} / \mathrm{S}$ 分析的稳 定性, 计算公式如下: 
$V_{n}^{*}=\frac{V_{s}(n)}{\sqrt{n}}$.

以 $\lg (n)$ 为横坐标, 以 $V_{n}{ }^{*}$ 为纵坐标绘制 $V_{n}{ }^{*}$ 统计量曲 线, 具有以下特征.

(1) 如果 $V_{n}$ * 统计量曲线向下倾斜, 则时间序列为 长程负相关特性, 此时 $0<H<0.5$.

(2) 如果 $V_{n}{ }^{*}$ 统计量曲线趋于水平, 则时间序列为 独立随机过程, 此时 $H=0.5$.

(3) 如果 $V_{n}{ }^{*}$ 统计量曲线向上倾斜, 则时间序列为 长程正相关特性, 此时 $0.5<H<1$.

\section{$3.3 \mathrm{~V} / \mathrm{S}$ 分析方法的短期相关性检验}

短期相关性特征为相邻变量之间的相关性会随着 滞后阶数的增大而迅速衰减. 短期相关性的存在可能 导致Hurst指数产生偏差, 进而影响长期相关特性. 为 降低短期相关性的影响，Peters ${ }^{[35,36]}$ 提出了利用 V/S 方 法分析序列的一阶自回归模型残差序列 $\mathrm{AR}(1)$, 并以 此检验 $\mathrm{V} / \mathrm{S}$ 分析方法对短期相关性的敏感度.

$\operatorname{AR}(1)$ 残差模型定义如下:

$\delta_{t}=\left(x_{t}-\bar{x}\right)-\omega_{1}\left(x_{t-1}-\bar{x}\right)$,

式中, $\delta_{t}$ 为序列的残差; $\bar{x}=\frac{1}{n} \sum_{t=1}^{n} x_{t}$ 为序列均值; $\omega_{1}$ 为序 列的自回归系数, 其值为序列的一阶自相关系数 $\rho$.

$\rho=\frac{\sum_{t=2}^{n} u_{t} u_{t-1}}{\sqrt{\sum_{t=2}^{n} u_{t}^{2}} \sqrt{\sum_{t=2}^{n} u_{t-1}^{2}}}$,

式中, $u_{t}(t=1,2, \ldots, n)$ 为数据序列的随机项.

\section{4 基于 $V / S$ 方法的中国电网停电事故时间间 隔序列的相关性分析}

\section{$4.1 \mathrm{~V} / \mathrm{S}$ 方法分析结果}

根据式(1) (4), 可求得中国电网停电事故时间间 隔序列的Hurst指数 $H$, 利用文献[34]中的Hurst指数显 著性检验方法, 可求得显著性检验统计量 $S$, 结果为 $S=2.0456, H=0.6791, R=0.9643, \mathrm{R} / \mathrm{S}$ 分析的计算结果为 $S=1.7676, H=0.7264, R=0.9912$. 同时可得: 利用最小二 乘法对 $\lg n$ 及 $\lg \left[V_{s}(n)\right]$ 进行线性拟合得到的相关系数 $R$, 如图5所示.

由上述可知, 经 $\mathrm{V} / \mathrm{S}$ 分析的中国电网停电事故时间
间隔序列的Hurst指数 $H$ 大于 0.5 , 且检验统计量 $S$ 大于 临界值 1.645 , 说明基于 $\mathrm{V} / \mathrm{S}$ 分析得到的Hurst指数为 0.6791 是显著的，结合Hurst指数的意义可知，中国电 网停电事故的时间间隔序列具有长程正相关特性, 这 与 $\mathrm{R} / \mathrm{S}$ 分析的结果一致.

\subsection{V/S 方法的稳定性检验分析}

由式(5)计算 $V_{n}{ }^{*}$ 统计量, 并绘制 $V_{n}{ }^{*}$ 统计量曲线, 如 图6所示.

由图6可知, 中国电网停电事故时间间隔序列的 $V_{n}{ }^{*}$ 统计量曲线的总体趋势是向上倾斜的, 表明中国电网停 电事故的时间间隔序列具有长程正相关特性，这与 $\mathrm{V} / \mathrm{S}$ 分析方法计算的Hurst指数在 $0.5 \sim 1$ 之间是一致的.

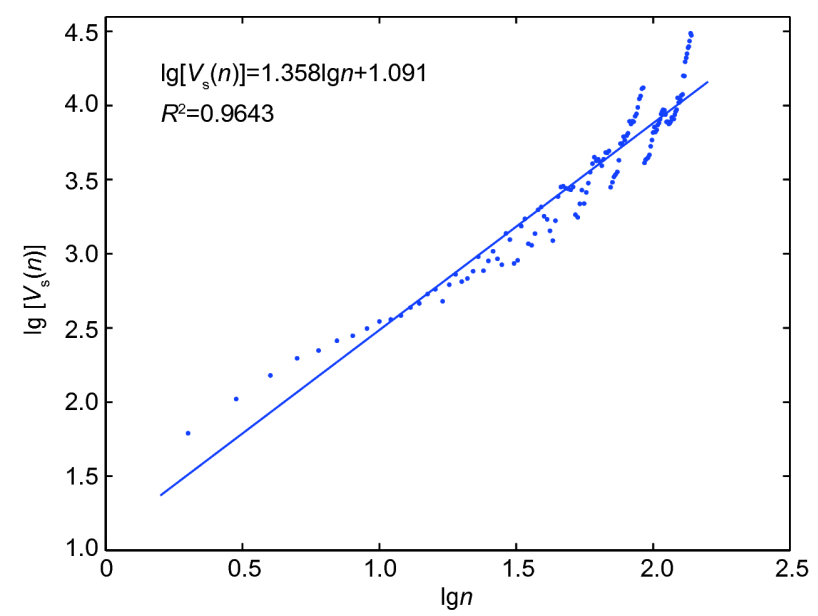

图 5 (网络版彩图) $\mathrm{V} / \mathrm{S}$ 方法计算的中国电网停电事故时间 间隔序列的Hurst指数

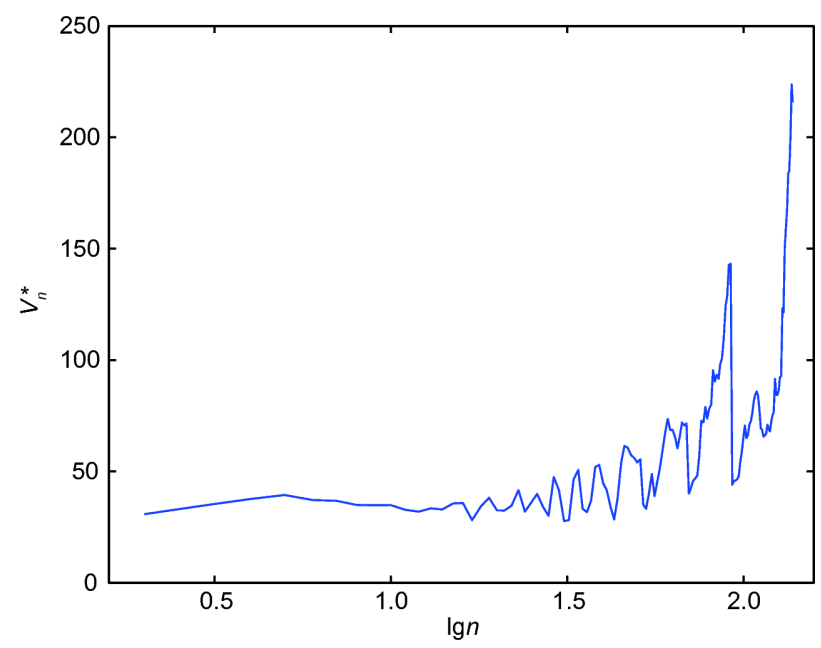

图 6 (网络版彩图) $V_{n}{ }^{*}$ 统计量曲线 


\subsection{V/S 方法的短期相关性检验分析}

根据式(6)计算中国电网停电事故时间间隔序列 的残差序列 $\mathrm{AR}(1)$, 利用 $\mathrm{V} / \mathrm{S}$ 方法对残差序列进行分析, 可得残差序列的Hurst指数为 0.6487 . 由残差序列的 Hurst指数大于 0.5 可知，中国电网停电事故的时间间 隔序列在去除短期相关性后仍存在长程正相关特性, 进一步验证了中国电网停电事故的时间间隔序列具有 长程正相关特性的分析结果是可靠的. 此外, 残差序列 的Hurst指数 0.6487 小于时间间隔序列的Hurst指数 0.6791, 表明时间间隔序列存在短期相关性, 且通过去 除短期相关性降低了Hurst指数偏离 0.5 的程度, 减弱了 时间间隔序列的相关性程度，但增加了 Hurst值的可信 度. 两者的Hurst指数相差并不大, 表明 $\mathrm{V} / \mathrm{S}$ 分析对于序 列的短期相关性的敏感程度较低，不会受序列本身存 在的短期相关性的影响而产生过大的偏差.

\section{5 电网停电事故时间间隔分布特性的意义}

通过对中国电网停电事故时间间隔的统计及 $V / S$ 分析，可知中国电网停电事故时间间隔的分布特性为 服从幂律分布，具有长程正相关性．这种分布特性具 有如下意义.

(1) 明确了中国电网停电事故时间间隔的分布规 律，该幕律分布表明电网停电事故在长时间间隔后仍 然有一定的发生概率，而这一概率是不可忽视的．据 此, 可为电网进行风险评估提供决策依据.

(2) 时间间隔是相邻两次停电事故之间的时间, 其 统计数据表明导致停电的发起事件(简称停电事件)的 性质, 对比美国电网停电事故时间间隔的指数分布, 可 知中国电网停电事故时间间隔的幂律分布及长程正相 关性表征了停电事件中存在着非随机的因素，这可为 预防大停电事故及模拟连锁故障提供参考.

(3) 电网停电事故时间间隔的幂律分布与文献 [12]研究的电网停电事故损失负荷的幂律分布既有联 系又有区别，两者都验证了中国电网的停电事故具有 自组织临界特性，但两者的研究对象及表征意义是不 同的. 前者研究的是电网停电事故的时间间隔序列, 其幕律分布表明了电网停电事故在长时间间隔后仍存 在不可忽视的发生概率，这是从时间角度对电网停电 事故发生概率的分析; 后者研究的是电网停电事故的 损失负荷序列，其幂律分布表明了电网发生大规模停
电的概率是不可忽视的, 这是对电网发生大规模停电 事故概率的分析. 两者是从不同的角度对中国电网停 电事故的内在动力学机理进行的不同方面的分析研 究, 具有同样重要的研究意义.

(4) 表明了中国电网停电事故的时间间隔序列具有 长程正相关性和统计自相似性, 这种正相关性说明电网 停电事故的时间间隔不是随机的, 表明中国未来电网发 生停电事故的时间间隔与过去发生的停电事故的时间 间隔存在相关性; 这种统计自相似性说明了中国电网 停电事故的时间间隔具有一定的可预测性 ${ }^{[37,38]}$.

(5) 电网停电事故的时间间隔与停电事故发生的 时间存在一一对应的关系, 由电网停电事故时间间隔 的非随机性, 可知电网停电事故发生的时间也具有非 随机性; 由电网停电事故时间间隔的可预测性，可知 电网停电事故发生的时间也具有可预测性，这就为构 建电网停电事故的预测模型提供了理论依据.

（6）电网停电事故时间间隔序列的长程正相关性 与文献[34]分析的电网停电事故损失负荷序列的长征 正相关性既有联系又有区别, 两者都验证了中国电网的 停电事故具有长程相关性, 但两者的研究对象及表征意 义是不同的. 前者研究的是电网停电事故的时间间隔序 列, 其长程相关性表明了电网停电事故的发生在时间上 不是随机的，而是具有相关性的，这是从时间角度对电 网停电事故发生规律的分析; 后者研究的是电网停电 事故的损失负荷序列，其长程相关性表明了电网发生 停电事故的损失负荷大小不是随机的，而是具有相关 性的，这是对电网发生停电事故的损失负荷大小的分 析，两者是从不同的角度对中国电网停电事故的不同 方面进行的分析研究, 旨在更全面地揭示中国电网停 电事故的复杂动态特性, 具有同样重要的研究意义.

(7) 能在一定程度上反映电力系统停电事故在时 间行为上的内在演化规律, 且以Hurst指数为 0.6791 度 量停电事故时间行为的相关性程度, 对研究电网停电 事故的内在动力学机理和预防大停电事故的发生具有 参考价值.

\section{6 讨论与结论}

本文统计分析了中国电网停电事故时间间隔序列 的分布特性，发现了中国电网停电事故的时间间隔序 列不满足指数分布, 而是近似满足幂律分布. 基于 $\mathrm{V} / \mathrm{S}$ 
方法分析了中国电网停电事故时间间隔序列的相关 性，指出了中国电网停电事故时间间隔序列具有长程 正相关性和自相似性. 中国电网停电事故时间间隔的 分布特性，表征了中国电网停电事故的发生在时间上 不是随机的而是长程相关的，且电网停电事故在长时 间间隔后仍存在不可忽视的发生概率，进一步揭示了 中国电网的复杂动态特性, 为电网停电事故的预测提 供了理论依据，并可为预防大停电事故的发生和评估
电网风险提供决策依据.

需要说明的是, 本文的主要工作在于利用历史统 计数据分析中国电网停电事故时间间隔的分布特性, 旨在揭示电网的复杂动态特性, 在分析中虽指出停电 事故发生时间的可预测性，但未分析其预测方法，在 下一步的研究中, 将展开深入的研究, 去探索电网停 电事故的预测模型，其结果可为电网进行风险管理提 供决策依据.

\section{参考文献}

1 郭剑波, 于群, 贺庆. 电力系统复杂性理论初探. 北京: 科学出版社, 2012. 149-150

2 胥威汀, 刘俊勇, 李旻, 等. 避免电网连锁解列的全局协调控制策略. 电力自动化设备, 2013, 33: 33-39

3 于群, 张敏, 曹娜, 等. 基于模糊元胞自动机的电网连锁故障控制策略. 电力自动化设备, 2016, 36: 90-95

4 曾辉, 孙峰, 李铁, 等. 澳大利亚“9.28”大停电事故分析及对中国启示. 电力系统自动化, 2017, 41: 1-6

5 Carreras B A, Newman D E, Dobson I, et al. Initial evidence for self-organized criticality in electric power blackouts. In: Proceedings of 33rd Annual Hawaii International Conference on System Science. Maui, 2000. 1-6

6 Chen J, Thorp J S, Parashar M. Analysis of electric power system disturbance data. In: Proceedings of 34th Hawaii International Conference on System Science. Maui, 2001. 738-744

7 Carreras B A, Newman D E, Dobson I, et al. Evidence for self-organized criticality in electric power blackouts. In: Proceedings of 34th Hawaii International Conference on System Science. Maui, 2001. 705-709

8 Carreras B A, Newman D E, Dobson I, et al. Evidence for self-organized criticality in a time series of electric power system blackouts. IEEE Trans Circuits Syst I, 2004, 51: 1733-1740

9 Hines P, Apt J, Talukdar S. Large blackouts in North America: Historical trends and policy implications. Energy Policy, 2009, 37: 5249-5259 Cornforth D. Long tails from the distribution of 23 years of electrical disturbance data. In: Proceedings of 2009 IEEE/PES Power Systems Conference and Exposition. Seattle, 2009. 1-8

11 Carreras B A, Newman D E, Dobson I. North American blackout time series statistics and implications for blackout risk. IEEE Trans Power Syst, 2016, 31: 4406-4414

12 于群, 郭剑波. 中国电网停电事故统计与自组织临界性特征. 电力系统自动化, 2006, 30: 16-21

13 于群, 郭剑波. 中国电力系统停电事故自组织临界性的研究. 电网技术, 2006, 30: 1-5

14 梅生伟, 薛安成, 张雪敏. 电力系统自组织临界特性与大电网安全. 北京: 清华大学出版社, 2009. 16-60

15 晁岱峰, 杨军选, 苏盛, 等. 养阳配电网故障的自组织临界性及其诱因. 电网技术, 2011, 35: 72-75

16 Boffetta G, Carbone V, Giuliani P, et al. Power laws in solar flares: Self-organized criticality or turbulence? Phys Rev Lett, 1999, 83: 4662-4665

17 Weron R, Simonsen I. Blackouts, risk, and fat-tailed distributions. In: Proceedings of 3rd Nikkei Econophysics Symposium. Tokyo, 2005. 215219

18 徐立新, 杨建美, 潮铸, 等. 电网故障时间间隔的统计分布及非线性特征分析. 电网技术, 2013, 37: 3482-3488

19 于群. 电力系统大停电的自组织临界特性研究. 中国电力科学研究院, 2010

20 国家电力调度通信中心. 全国电网典型事故分析(1988 1998). 北京: 中国电力出版社, 2000

国家电力调度通信中心. 全国电网典型事故分析(1999 2007). 北京: 中国电力出版社, 2008

国家电网公司安全监察部. 国家电网公司2007年安全生产事故报告. 北京: 中国电力出版社, 2007

国家电网公司安全监察部. 国家电网公司2008年安全生产事故报告. 北京: 中国电力出版社, 2009

国家电网公司安全监察部. 国家电网公司2009年安全生产事故报告. 北京: 中国电力出版社, 2010

国家电网公司安全监察部. 国家电网公司2010年安全生产事故报告. 北京: 中国电力出版社, 2011

国家电网公司安全监察质量部. 国家电网公司2011年安全生产事故报告. 北京: 中国电力出版社, 2012 
国家电网公司安全监察质量部. 国家电网公司2012年安全生产事故报告. 北京: 中国电力出版社, 2013

国家电网公司安全监察质量部. 国家电网公司2013年安全生产事故报告. 北京: 中国电力出版社, 2014

29 国家电网公司安全监察质量部. 国家电网公司2014年安全生产事故报告. 北京: 中国电力出版社, 2015

30 Clauset A, Shalizi C R, Newman M E J. Power-law distributions in empirical data. SIAM Rev, 2009, 51: 661-703

31 何兴强, 李仲飞. 上证股市收益的长期记忆: 基于 V/S的经验分析. 系统工程理论与实践, 2006, 26: 47-54

乔美英, 陈金金, 兰建义. 基于V/S分析的瓦斯涌出量分形特性研究. 中国煤炭, 2014, 40: 104-110

294

34 于群, 屈玉清, 石良. 基于相对值法和Hurst指数的电网停电事故自相关性分析. 电力系统自动化, 2018, 42: 55-60

35 Peters E E. Fractal Market Analysis. New York: John Wiley \& Sons, Inc., 1994

\title{
Statistics and distribution characteristics analysis of time interval of blackout in Chinese power grid
}

\author{
YU Qun ${ }^{1}$, QU YuQing ${ }^{1}, \mathrm{CAO} \mathrm{Na}^{1} \&$ YI Jun ${ }^{2}$ \\ ${ }^{1}$ College of Electrical Engineering and Automation, Shandong University of Science and Technology, Qingdao 266590, China; \\ ${ }^{2}$ China Electric Power Research Institute of Power Systems, Beijing 100085, China
}

To study the complex dynamic characteristics of blackouts in Chinese power grid, the distribution characteristics of time intervals of blackouts in Chinese power grid are researched. Firstly, the time intervals of blackouts in Chinese power grid from 1981-2014 are analyzed by statistical analysis and normality test. It is proved that the time intervals of blackouts in Chinese power grid approximately satisfies the power-law distribution. Secondly, utilizing the V/S method, the correlation of time intervals of blackouts in Chinese power grid is researched. It is proved that the time intervals of blackouts in Chinese power grid have the strong positive correlation and self-similarity on long time scale. Finally, the significance of distribution characteristics of time intervals of blackouts in Chinese power grid is given.

blackout, time interval, power law distribution, V/S method, correlation, distribution characteristics doi: $10.1360 /$ N092018-00103 